\title{
Firing Properties of Spinal Interneurons during Voluntary Movement. II. Interactions between Spinal Neurons
}

\author{
Yifat Prut ${ }^{2}$ and Steve I. Perlmutter ${ }^{1}$ \\ ${ }^{1}$ Department of Physiology and Biophysics and the Washington National Primate Research Center, University of Washington, Seattle, Washington 98195, \\ and ${ }^{2}$ The Hebrew University, Hadassah Medical School, Jerusalem 91120, Israel
}

The relationship between the activity of pairs of simultaneously recorded spinal interneurons (INs) in the cervical enlargement was studied in five monkeys performing voluntary wrist movements. The tendency for INs to exhibit similar response properties and synchronized firing was tested as a function of physical distance between the cells and their correlational linkages with forearm muscles. Nearby INs tended to have more similar torque and direction turning (signal correlation) and more similar response profiles (e.g., tonic vs phasic firing) than INs that were far apart. This suggests that nearby cells receive common synaptic input. In contrast, the trial-to-trial covariation of rate around the mean rate for all trials (noise correlation) was independent of the distance between the neurons. Furthermore, signal and noise correlation were independent, suggesting different underlying mechanisms. Surprisingly, spike-to-spike correlation between INs was relatively infrequent and weak, as measured by cross-correlation histograms. In contrast, single motor units (SMUs) in forearm muscles fired more synchronously, particularly for SMUs in single extensor muscles. Either common drive to INs is too weak to induce synchronized firing, or there is an active decorrelation mechanism within IN networks.

Key words: spinal cord; interneurons; primates; firing pattern; synchronization; motor control

\section{Introduction}

Spinal interneurons (INs) affect motoneuron (MN) firing through their activity and connectivity patterns, which have been well studied. In the companion paper (Prut and Perlmutter, 2003), we argued that the spike-to-spike firing properties of single INs also may contribute to this integration. In addition, network interactions between INs may modulate the transmission of information through spinal pathways and may help shape muscle activity.

The most widely studied form of network interaction is the synchronous firing of neurons. Synchrony has been described throughout the motor system, although its function is somewhat controversial. Correlated firing in M1 may dynamically couple the activity of functionally related neurons (Vaadia et al., 1995; Riehle et al., 1997; Grammont and Riehle, 1999), code movement parameters (Hatsopoulos et al., 1998; Maynard et al., 1999), link the activation of groups of muscles (Smith and Fetz, 1989; Jackson et al., 2003), be related to levels of attention or arousal (Murthy and Fetz, 1996), or increase the efficiency with which motoneurons are recruited (Baker et al., 2001). In contrast, others consider such correlations to be redundant representa-

\footnotetext{
Received March 31, 2003; revised July 30, 2003; accepted Aug. 19, 2003.

This work was conducted at the Washington National Primate Research Center, University of Washington, and was supported by National Institutes of Health Grants NS36781, NS12542, and RR00166, the United States-Israel Binational Science Foundation, and the Israel Science Foundation. We thank Larry Shupe and Jonathan Garlid for technical support and Moshe Abeles and Eberhard Fetz for valuable comments on this manuscript.

Correspondence should be addressed to Yifat Prut, Department of Physiology, The Hebrew University, Hadassah Medical School, P.0. Box 12272, Jerusalem 91120, Israel. E-mail: yifat@md.huji.ac.il.

Copyright $\odot 2003$ Society for Neuroscience $\quad$ 0270-6474/03/239611-09\$15.00/0
}

tions of movement-related information (Lee et al., 1998; Oram et al., 2001).

Correlated activity is expected in the spinal cord given the divergent anatomy of both descending and sensory inputs (Windhorst, 1988). Synchronous discharge of MNs has been widely reported (Sears and Stagg, 1976) (for review, see Farmer et al., 1997). Two lines of evidence suggest that branching corticospinal axons underlie this phenomenon. First, synchrony is altered in patients with stroke, spinal cord injury, or amyotrophic lateral sclerosis (Datta et al., 1991; Schmied et al., 1999; Smith et al., 1999) but not in subjects with severe peripheral deafferentation (Baker et al., 1988; Schmied et al., 1999). Second, synchrony appears to be under some degree of voluntary control (Schmied et al., 1993). Nonetheless, the extent to which INs contribute to MN synchronization is unknown.

Only limited data are available on correlated firing between INs. Dorsal horn cells with overlapping peripheral receptive fields are synchronized (Brown et al., 1979; Eblen-Zajjur and Sandkuhler, 1997), probably because of branching afferent fibers. Synchrony among intermediate laminae INs is suggested by features in spike-triggered averages of muscle activity that exhibit a facilitation or suppression that straddles the trigger time (Perlmutter et al., 1998). Synchronous firing may facilitate a rapid grouping and regrouping of INs that participate in different motor tasks, although there is currently no direct evidence in support of this hypothesis.

In this study, we searched for evidence of common synaptic input to INs and directly measured the extent of correlated firing by simultaneously recording the activity of pairs of INs. Direct measures of network interactions in the cord may reveal mechanisms by which specific motor patterns are selected. 


\section{Materials and Methods}

The activity of pairs of spinal neurons was recorded simultaneously with one or two glass-coated tungsten electrodes in macaque monkeys performing wrist movements. The two electrodes were advanced into the spinal cord at different mediolateral positions but at the same rostrocaudal level, so that each pair consisted of two neurons in the same spinal segment. The distance between two neurons recorded on different electrodes was not reconstructed systematically but was estimated as the distance between the electrode tips when positioned at the recording sites. Ninety percent of the pairs were between 1.4 and $3.9 \mathrm{~mm}$ apart, with a median distance of $2.4 \mathrm{~mm}$. Neurons recorded by the same electrode were considered to be located near each other, and those recorded by two different electrodes were taken to be far apart.

The behavioral tasks and data acquisition protocols are described in the companion paper (Prut and Perlmutter, 2003). In short, monkeys generated flexion and extension torques at the wrist to track visual targets on a video monitor. Monkey B exerted alternating flexion and extension torques. Monkey W performed a center-out task in which flexion and extension movements were initiated from rest (zero torque exerted at the wrist). Monkeys $\mathrm{E}, \mathrm{F}$, and $\mathrm{H}$ performed a delayed response task in which the upcoming movement was visually cued $1-2$ sec before a second visual signal instructed the monkey to execute the movement. For monkey $\mathrm{H}$, visual and proprioceptive cues were presented in alternating blocks of trials.

\section{Single-motor unit recording}

In one monkey $(\mathrm{H})$, we recorded the activity of single motor unit (SMUs) in forearm muscles. Multiple pairs of wires $(25 \mu \mathrm{m}$ single-stranded, stainless steel) were implanted in the same and different muscles before 11 recording sessions on different days. Targeted muscles were identified using surface landmarks, twitches elicited by electrical stimulation through the wire, or both. Electrical potentials were continuously digitized and stored on disk. SMUs were extracted off-line using a spike sorting program based on a principle component analysis (AlphaSort; Alpha Omega, Nazareth, Israel). Well isolated, stationary SMUs were defined using the criteria described for single INs in the companion paper (Prut and Perlmutter, 2003). No distinction was made between nearby and distant SMUs because the muscle fibers of motor units can be distributed widely within a muscle.

\section{Data analysis}

The companion paper (Prut and Perlmutter, 2003) describes methods for identifying MNs and INs with postspike or synchrony effects in forearm muscles using spike-triggered averages of electromyographic (EMG) activity and for identifying task-related modulation of activity on a trialby-trial basis. The following analyses were performed to characterize the extent to which pairs of INs received common synaptic input. For these analyses, we included data in which one or both of the recorded cells had no more than $1 \%$ of spikes with interspike intervals of $<1 \mathrm{msec}$ (i.e., no absolute refractory period), indicating contamination from the spikes of another neuron. Although eliminating recordings even with this amount of contamination is important when characterizing the firing regularity of neurons (Prut and Perlmutter, 2003), it is less critical for identifying common synaptic input.

Clustering of response histograms. Typical response profiles of neuronal activity during generation of torque were identified for three monkeys using a cluster analysis. Response histograms for each IN, aligned on torque onset, were computed separately for all flexion and extension trials (e.g., see Fig. 7C-E, which shows responses of single motor units). Each histogram was treated as a vector in $n$-dimensional space, where $n$ is the number of bins in the histogram, and was normalized by dividing by its norm. Response histograms for monkey $\mathrm{W}$ and for monkeys $\mathrm{E}$ and $\mathrm{F}$ combined (because they performed the same task) were grouped separately using a K-mean clustering algorithm (Hartigan, 1975) with a predefined number of clusters (three or four; see Results). The robustness of the clustering was estimated by running the algorithm several hundred times to determine the most reproducible set of clusters. We did not pursue the significance level of these clusters because the main purpose was simply a categorization of the responses. Using the most reproduc- ible set of clusters, the response of each neuron was assigned to the cluster from which it had the smallest distance, $D$, defined post hoc as the following Euclidean metric:

$$
D=\sqrt{\sum_{\mathrm{i}}\left(r_{\mathrm{i}}^{\mathrm{k}}-c_{\mathrm{i}}^{\mathrm{j}}\right)^{2}},
$$

where $r$ is the $k$ th neuron's vector, (i.e., response histogram); $c$ is the vector of the $j$ th cluster center (i.e., the average response histogram of the vectors forming a cluster); and $i$ is the bin index.

The clustering analysis was performed only for neurons that exhibited significant task-related modulation of activity. The choice of a specific criterion for modulated activity and of specific parameters for the response histograms (i.e., bin size and smoothing) did not significantly affect the results.

Signal and noise correlation between pairs of INs. Signal and noise correlation were computed for pairs of simultaneously recorded cells from monkeys W, E, F, and B using methods similar to those of Lee et al. (1998) and Bair et al. (2001) for cortical neurons. These monkeys generated ramp-and-hold torques to seven different target levels (three in flexion, three in extension, and zero torque during the rest period). Signal and noise correlation were calculated between INs that each had torque selectivity, direction selectivity, or both.

Direction-sensitive cells were identified as those neurons with significantly different average firing rates in the active-hold period of single flexion and extension trials ( $t$ test between the distribution of single-trial rates, $p<0.05$; minimum five flexion and five extension trials). Torque sensitivity was determined by computing the regression coefficient between average firing rate and torque level during the active-hold periods (flexion and extension trials treated separately). INs were classified as exhibiting torque sensitivity when one or both regression coefficients were significantly different from zero $(p<0.05$; minimum 10 flexion and 10 extension trials).

Signal correlation was a measure of the similarity between the torque or direction selectivity of two neurons. It was computed for pairs of INs for which there were data from at least four torque levels and at least four trials for each level. The average firing rates during the active-hold period in single trials were averaged separately for each torque level and used as the elements of an $n$-dimensional vector ( $n$ between 4 and 7), referred to as the signal vector of the neuron. Signal correlation was defined for each pair of INs as the correlation coefficient of their signal vectors.

Noise correlation was a measure of the trial-to-trial covariation of rate between two simultaneously recorded neurons, independent of any direction- or torque-related effects on firing. For each neuron, the mean and SD of discharge rate during the active-hold period across all trials of a given type (i.e., at a given torque level for torque-sensitive cells or in a given torque direction for direction-sensitive cells) were calculated for all trial types. Then the average rate in single trials was normalized by subtracting the mean rate across all trials of the same type and dividing by the $\mathrm{SD}$ across all trials of the same type. An $m$-dimensional vector, termed the noise vector of the neuron, was created using the normalized rates for the $m$ trials in which both neurons were recorded. Noise correlation was defined for each pair of neurons as the correlation coefficient between their noise vectors. The normalization procedure eliminated correlations attributable to similar task-related modulation of rate.

Cross-correlation of firing between pairs of INs. The spike-to-spike correlation of firing between pairs of INs and pairs of SMUs was determined using cross-correlogram histograms (XCRs), as described previously (Perkel et al., 1967; Abeles, 1991). Cross-correlations with $1 \mathrm{msec}$ bins were computed for $\pm 150 \mathrm{msec}$ around the trigger spike and were smoothed using a Gaussian with an SD of 1 or $2 \mathrm{msec}$. Only XCRs for which the mean counts per bin were $\geq 15$ were analyzed.

XCRs were computed separately for the rest period, the active-hold period for flexion torques, and the active-hold period for extension torques. The mean baseline level of the XCR was calculated using the last $50 \mathrm{msec}$ in both negative and positive lags, and the $99 \%$ confidence intervals were computed around this value. Central peaks were defined as near-zero events that crossed the upper confidence limit for at least two 

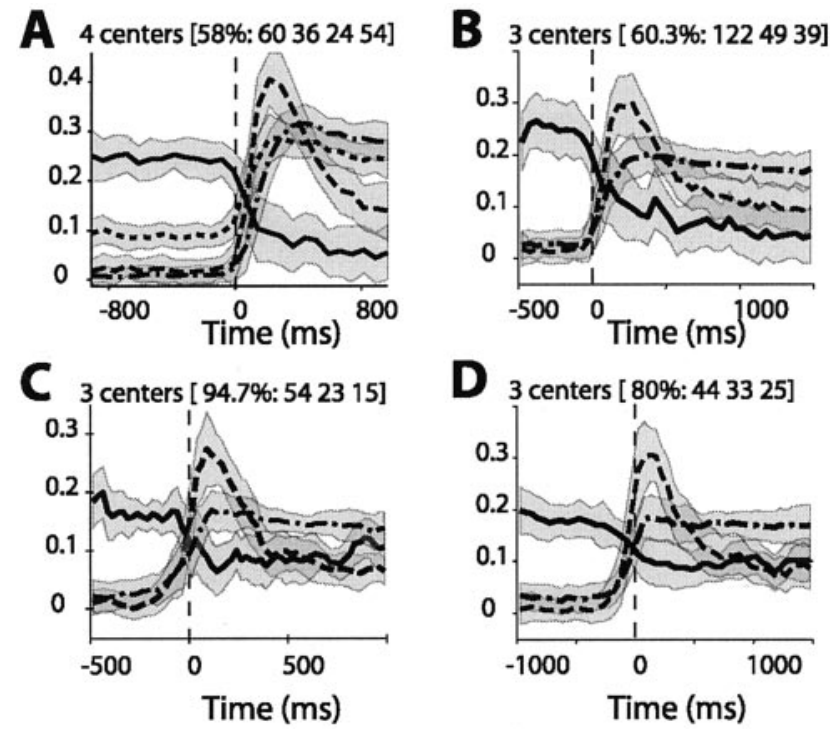

Figure 1. Cluster centers (lines) and dispersion (shading) for four separate analyses. The center was taken as the median of all vectors (i.e., normalized response histograms) constituting a cluster, and the dispersion was estimated as \pm 1 median absolute deviation from the median. $A, B$, Results of clustering of the data from monkey $W$ using different clustering parameters. $C, D$, Results of clustering of the data from monkeys $F$ and $E$ combined using different clustering parameters. The numbers above each plot give the frequency of occurrence of the centers for 300 repetitions of the clustering and the number of neurons in each of the clusters (in order, for the dashed and dotted, dashed, solid, and, in A, dotted, lines). Units on the $y$-axis are normalized firing rates; movement onset occurs at time zero.

consecutive bins. The onset and offset of each peak were taken as the first and last crossings of the mean baseline level.

\section{Results}

\section{Similarity of response profile}

The tendency for nearby cells to exhibit similar response profiles was estimated for 298 INs using the K-mean clustering analysis. Grouping of response histograms for monkeys $\mathrm{E}$ and $\mathrm{F}$ gave reproducible cluster centers when three clusters were used (Fig. $1 C, D)$. Repeated runs of the algorithm (see Materials and Methods) with four clusters converged on many different centers, each repeating only a few times. For monkey W, three clusters also gave more reproducible results when response histograms with short premovement periods $(500 \mathrm{msec}$ ) were analyzed (Fig. $1 \mathrm{~B}$ ). However, for histograms with longer premovement periods (1000 $\mathrm{msec})$, four centers provided more reproducible results (Fig. 1A).

The shape and frequency of the cluster centers were similar for monkeys performing the flexion-extension task without (monkey W) and with (monkeys E and F) an instructed delay period. Figure 1 presents cluster centers from two different analyses, using different clustering parameters, of the data from monkey $\mathrm{W}$ (Fig. $1 A, B$ ) and two analyses of the data from monkeys $\mathrm{E}$ and $\mathrm{F}$ (Fig. 1C,D). The cluster centers correspond to phasic (dashed line), tonic (dashed-dotted line), inhibitory (solid line), and baseline-offset tonic (dotted line) responses. The fourth response type (dotted line) was probably not seen for monkeys $\mathrm{E}$ and $\mathrm{F}$ because of the delay task that they performed. Many INs are inhibited during an instructed delay period (Prut and Fetz, 1999), and this inhibition would tend to mask any premovement elevation in firing rate. Each of the clusters shown in Figure 1 was present in $>50 \%$ of 300 separate runs of the clustering algorithm. The tonic response type was the most common, and the inhibitory response was seen least frequently.
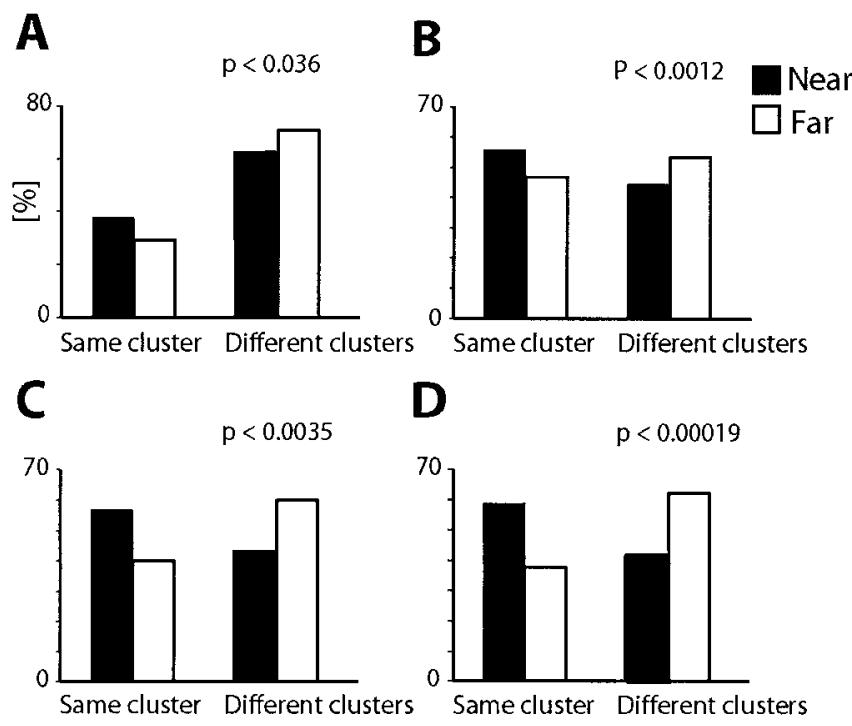

Figure 2. Response similarity of nearby versus distant INs. The percentage of pairs of simultaneously recorded neurons whose response histograms belonged to the same or different clusters for nearby (black bars) and distant pairs (white bars) is shown for the four analyses of Figure 1. The $p$ values show significance levels of $\chi^{2}$ tests for dependence of cluster similarity on interneuron distance.

Nearby neurons were more likely than distant cells to exhibit similar movement-related response profiles. As shown in Figure 2 , neurons recorded from the same electrode had response histograms that tended to belong to the same response cluster more often than neurons recorded from different electrodes [for each plot, the black bar is higher than the white bar on the left (Same cluster), but the black bar is lower than the white bar on the right (Different cluster)]. This difference was statistically significant $\left(\chi^{2}\right.$ test $)$ for all repeatable clusters derived from data for monkeys $\mathrm{W}, \mathrm{E}$, and $\mathrm{F}$.

Relationship between signal correlation and noise correlation The clustering of response histograms characterizes the similarity in temporal profile of the activity of neurons during movement. In contrast, the signal correlation for a pair of neurons quantifies their similarity in response tuning for direction and torque. Noise correlation is a measure of the trial-to-trial covariation of their firing rates around the mean values. Signal and noise correlations were calculated for 204 pairs of INs.

There was no clear relationship between signal and noise correlation, as seen in the examples shown in Figure 3. The pairs in Figure $3, A$ and $C$, had strong positively and negatively correlated signal vectors, respectively, but low noise correlation. The pair in Figure $3 B$ had low signal correlation but high noise correlation.

The independence of signal and noise correlation can be seen for all pairs in Figure 4. The marginal distribution of the correlation coefficients for the signal vectors exhibits a bias toward high values. On the other hand, the correlation coefficients of the noise covariation are distributed almost symmetrically around zero (the mean level was 0.035 , which was significantly different from zero; $p<0.05)$. The scatterplot demonstrates that the extent to which the signal vectors of two neurons were correlated did not predict the extent of their noise correlation. Furthermore, the pairs with significant, positive noise correlation (Fig. 4, white symbols) appear uniformly distributed along the $x$-axis, showing no tendency for a particular level of signal correlation.

Pairs of INs exhibited distance-dependent signal correlation 
A

\section{Signal correlation}

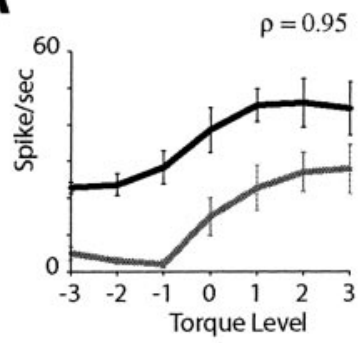

B

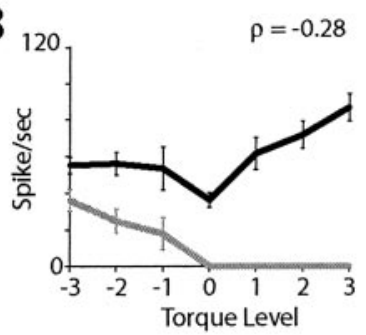

C

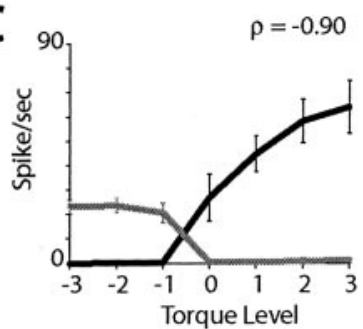

Noise correlation
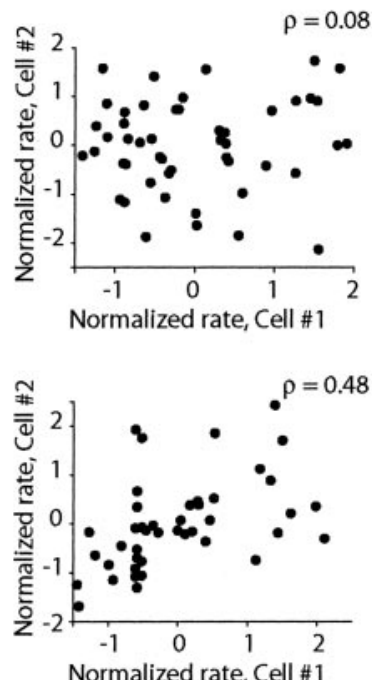

Normalized rate, Cell \#1

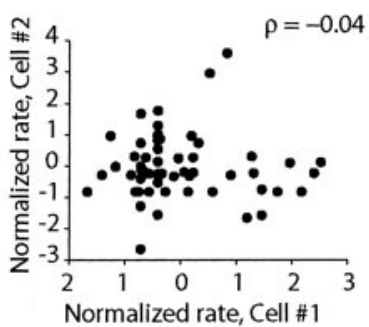

Figure 3. Signal and noise correlations for three pairs $(A-C)$ of INs recorded by the same electrode. Left, Signal vectors (firing rate as a function of torque level) with error bars indicating SD; correlation coefficients between the vectors (signal correlation) are shown above each plot. Right, Scatterplot of the single trial noise values (see Materials and Methods) for the two neurons, arbitrarily designated as cell \#1 and cell \#2; correlation coefficients (noise correlation) are shown above each plot.

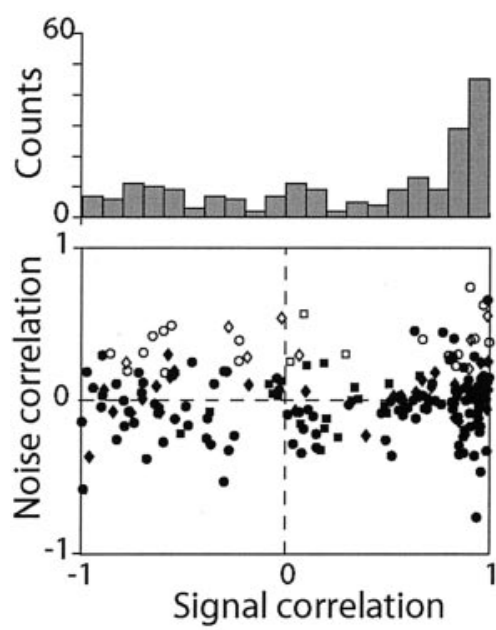

$\mathrm{N}=204$

- Torque sensitive

- Torque+Direction sensitivity

- Direction sensitivity

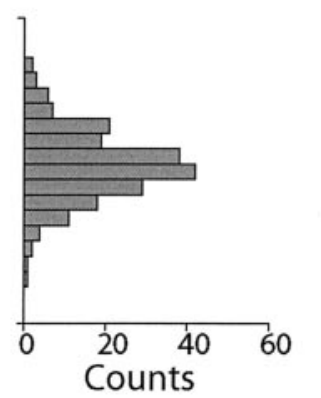

Figure 4. Relationship between signal and noise correlations for 204 pairs of INs with torque (squares), direction (circles), or torque and direction (diamonds) sensitivity. The marginal distributions are shown as histograms above and to the right of the scatterplot. White symbols indicate positive values of noise correlation significantly different from zero.

but had distance-independent noise correlation. Figure 5, $B$ and $E$, shows that neurons recorded from the same electrode were more likely to exhibit stronger signal correlation than neurons recorded from different electrodes. Correlation coefficients found
Same electrode

$(n=158)$

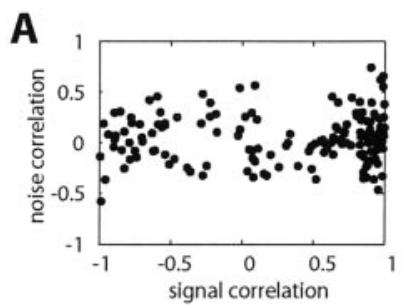

B
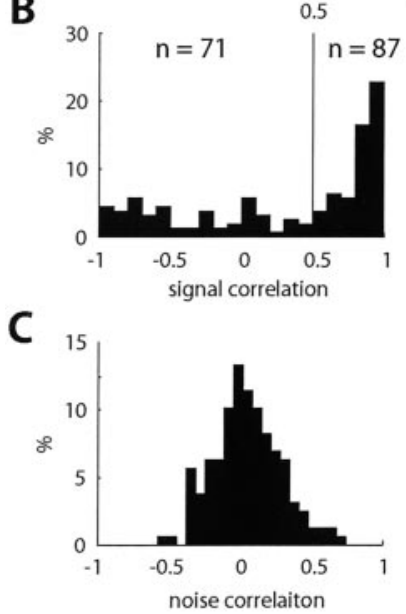

Neighboring electrodes ( $n=46)$
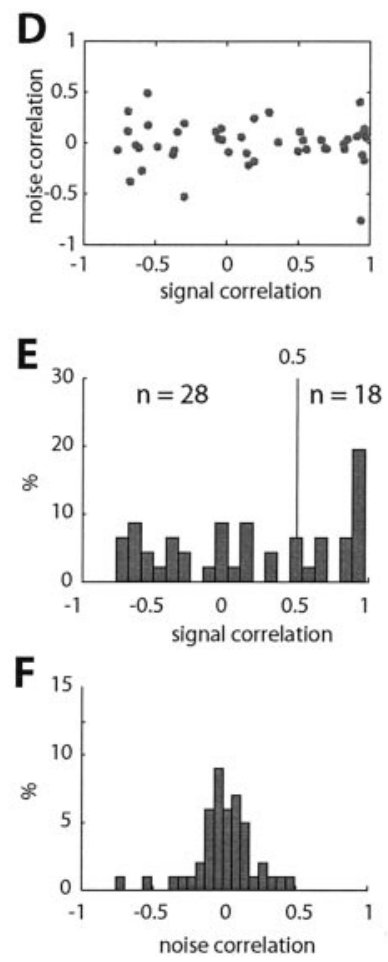

Figure 5. Distance dependency of signal correlation and noise correlation. Scatter plots show noise versus signal correlations $(A, D)$ and distributions of signal $(B, E)$ and noise $(C, F)$ correlations for IN pairs recorded by the same electrode (left) or different electrodes (right).

for nearby cells were significantly larger than those found for distant cells (Kolmogorov-Smirnov test, $p<0.05$ ). Just as neurons located in close proximity had more similar response profiles than those located far apart, they were more likely to have similar response tuning for movement direction and torque amplitude.

In contrast, the distributions of the noise correlation coefficients were not significantly different (Kolmogorov-Smirnov test, $p>0.1$ ) for pairs of INs recorded from the same or different electrodes (Fig. 5C,F), although there was a small difference in the mean values $(0.046$ and -0.003 , respectively). Furthermore, the relationship between signal and noise correlation appeared independent of the distance between the neurons (Fig. 5A,D). These results differ from those reported for pairs of cortical neurons in M1 (Lee et al., 1998) and MT (Bair et al., 2001).

\section{Spike-to-spike correlation of INs}

Few IN pairs exhibited synchronized activity, and the extent of correlated firing was very weak even in those that did (Fig. 6). XCRs were analyzed for 188 pairs of INs, 116 (62\%) of which were recorded from the same electrode and $72(38 \%)$ of which were recorded from two different electrodes. Only 26 (13.8\%) pairs, all recorded from the same electrode, had a significant near-zero peak (Table 1), and almost all of these were of small amplitude. The paucity of synchronized firing in our population of INs is evidenced by the examples in Figure 6, $A$ and $B$, which present the best XCR peaks from our data set. In a few cases, XCRs exhibited oscillatory features centered around the trigger time.

The strength of XCR peaks for the 26 correlated pairs usually varied from task epoch to epoch. The IN of Figure $6 \mathrm{~A}$ exhibited correlated activity only during the active-hold period for flexion 
A Rest

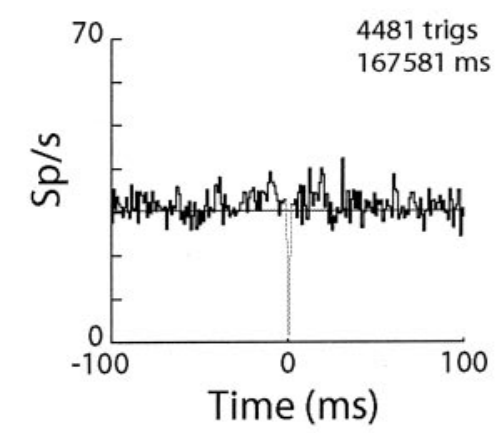

B

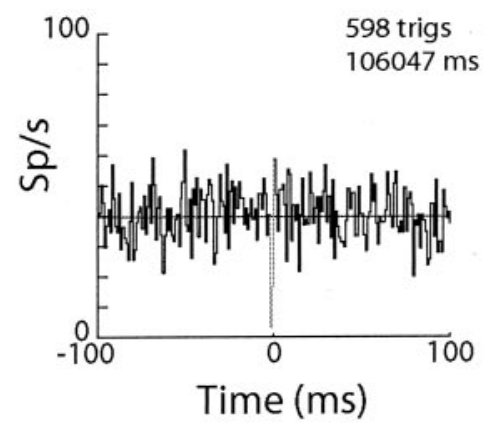

Active-hold, extension
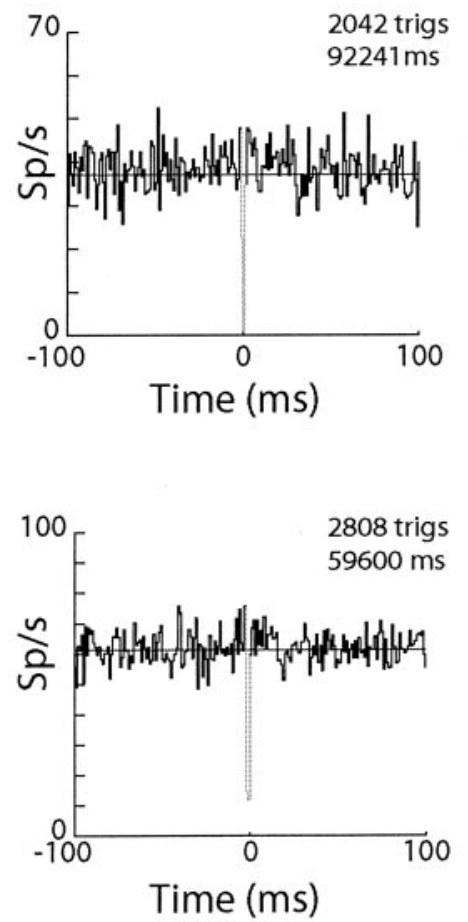

Active-hold, flexion
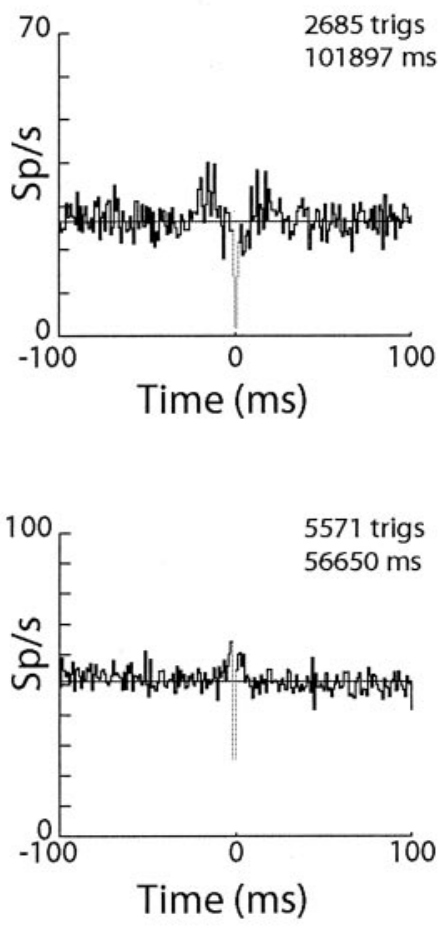

Figure 6. XCRs for two pairs of INs $(A, B)$ computed separately for the rest period (left) and active-hold periods for extension (center) and flexion (right) torques. Pairs were recorded by the same electrode. The zero-count bins around the trigger time (gray) are artifacts attributable to our inability to detect waveforms created by the superposition of the action potentials of the neurons. The number of trigger spikes (trigs) in the XCRs and the total time of recording in each epoch are shown above each plot.

Table 1. Relationships of correlated firing to behavioral epoch

\begin{tabular}{|c|c|c|c|c|c|}
\hline Epoch data & $\begin{array}{l}\text { Total } \\
\text { pairs }\end{array}$ & $\begin{array}{l}\text { Correlated } \\
\text { pairs }^{b}\end{array}$ & $\begin{array}{l}\text { Correlated } \\
\text { in } 1 \text { epoch }\end{array}$ & $\begin{array}{l}\text { Correlated } \\
\text { in } 2 \text { epochs }\end{array}$ & $\begin{array}{l}\text { Correlated } \\
\text { in } 3 \text { epochs }\end{array}$ \\
\hline \multicolumn{6}{|c|}{ Task dependency of correlated activity } \\
\hline \multicolumn{6}{|c|}{ Valid epochs/pair ${ }^{a}$} \\
\hline 1 & 97 & 8 & 8 & & \\
\hline 2 & 33 & 3 & 1 & 2 & \\
\hline 3 & 58 & 15 & 11 & 3 & 1 \\
\hline \multirow{2}{*}{\multicolumn{6}{|c|}{$\begin{array}{l}\text { Correlated activity as a function of epoch } \\
\text { Epoch }\end{array}$}} \\
\hline & & & & & \\
\hline Rest & 93 & 8 & & & \\
\hline Flexion active-hold & 116 & 16 & & & \\
\hline Extension active-hold & 128 & 9 & & & \\
\hline
\end{tabular}

${ }^{a}$ Number of epochs for which the $X C R$ had mean counts per bin of $\geq 15$.

${ }^{b}$ Number of pairs for which activity was correlated in one or more epochs.

torques. The pair in Figure $6 B$ fired synchronously during maintained flexion and extension torques, but the central peak was more pronounced during flexion. Significant peaks were present for 18 pairs of INs for which there were sufficient data to compute XCRs in more than one behavioral epoch (Table 1). Of these, only three pairs exhibited correlated activity in all analyzed epochs. There was a slight although nonsignificant $(p \geq 0.082)$ tendency for XCR peaks to occur during the active-hold period for flexion torque (Table 1). Thus, the small amount of correlated firing between INs exhibited strong behavioral dependence.

Although correlated firing was not a prominent feature of IN activity, it was of interest to compare the relationship between the synchronization that was present and linkages with muscles. Correlational linkages between cells and muscles were quantified using spike-triggered averages of EMG recordings in 162 of the 188 pairs for which XCRs were calculated (Table 2). There was no statistically significant tendency for pairs with or without XCR peaks to have correlational linkages with muscles $\left(\chi^{2}, p>0.25 ; 16\right.$ of 23 correlated pairs and 77 of 139 uncorrelated pairs exhibited postspike effects, synchrony effects, or both in muscles). In addition, INs that exhibited synchrony effects were no more likely to be correlated with the paired neuron than INs with postspike effects (Table 2). Among the 16 correlated pairs in which at least one neuron exhibited a correlational linkage with muscles, 12 pairs had XCR peaks that were present only during the behavioral epoch in which the muscles with postspike or synchrony effects were active.

Another way to probe the relationship between correlated activity and generation of muscle activity is to focus on pairs of INs that both have correlational linkages with coactive muscles (i.e., synergists and bidirectionally active muscles such as brachioradialis and pronator teres). We recorded from 16 such pairs, 8 of which exhibited correlated firing during at least one epoch of the task. Six of these correlated pairs had XCR peaks only during the behavioral epoch in which the muscles with postspike or synchrony effects were active (e.g., both neurons had postspike effects on extensor muscles, and their activity was correlated only during the active-hold period for extension torques).

Previous studies reported that signal, noise, and spike-tospike correlations were tightly linked for pairs of cortical neurons 
(Lee et al., 1998; Bair et al., 2001). No such relationship was found for INs. Table 3 shows that the strengths of signal and noise correlations were not significantly different for pairs of INs with or without XCR peaks (Kolmogorov-Smirnov tests, in all cases $p>0.1)$. This result, combined with our finding of no consistent relationship between signal and noise correlations (Fig. 4), suggests that these three measures of neuronal interaction are independent for INs.

\section{Spike-to-spike correlation of SMUs}

Correlated firing between motoneurons was investigated with simultaneous recordings of multiple SMUs from one or two muscles (Fig. 7A). SMUs had relatively low, periodic activity and discharged only for torques in one direction (Fig. $7 B, C$ ). XCRs were calculated for 122 pairs of SMUs (Table 4), of which 37 pairs (30.3\%) exhibited central peaks (Fig. 8). A significantly larger percentage of SMUs in extensor muscles were correlated compared with SMUs in flexor muscles (36 vs $12 \%$; $\chi^{2}$ test, $\left.p<0.01\right)$. This contrasts with the slight tendency for INs to be more correlated during active flexion. Pairs of SMUs recorded from the same muscle were more likely to be correlated than pairs recorded from different, coactive muscles (Table 4).

The strength of spike-to-spike correlations between pairs of INs and pairs of SMUs was compared using three (related) parameters: the duration of the XCR peak at its base (i.e., interval from peak onset to peak offset), the duration of the XCR peak at midheight (midway between mean and maximum values of the XCR), and the area of the peak (area above mean baseline level divided by area below mean baseline level between onset and offset times). The distribution of both duration parameters was similar for XCR peaks for SMUs and INs (Fig. 9A,B). However, the magnitude of the SMU peaks was significantly larger than that for INs (Fig. 9C). In part, this reflects the higher firing rate of INs than SMUs. Nevertheless, the results suggest a greater tendency for correlated activity between motoneurons than between INs (KolmogorovSmirnov, $p<0.01)$.

\section{Discussion}

\section{Common input to pairs of INs}

Nearby INs had similar response properties. Temporal profiles of activity and torque and direction tuning were more similar for neurons recorded from the same electrode than for neurons recorded from different electrodes. This suggests that neurons located close together receive common input, probably mediated by the divergent branching of descending (Shinoda et al., 1981; Li and Martin, 2002) and afferent (Ishizuka et al., 1979; Brown, 1981; Ralston et al., 1984) axons to the spinal cord.

The magnitude and distance dependence of the signal correlation between pairs of INs were similar to those reported for cerebral cortical neurons. Noise correlation between INs also was similar to that between cells in the primary motor cortex (Lee et al., 1998), although it was much weaker than for pairs in cortical
Table 2. Relationship of correlated firing to correlational linkages with muscles

\begin{tabular}{lll}
\hline STA effect $^{a}$ & Number of uncorrelated pairs & Number of correlated pairs \\
\hline $\mathrm{P}$ & 34 & 6 \\
$\mathrm{~S}$ & 33 & 5 \\
$\mathrm{P}+\mathrm{S}$ & 14 & 5 \\
Total & $77^{b}$ & 16 \\
\hline
\end{tabular}

Data are XCR of IN pairs for which at least one neuron had a correlational linkage with muscles. STA, Spike-triggered average; $P$, postspike effect; $S$, synchrony effect.

${ }^{a}$ STA effect for the two INs of the pair.

${ }^{b}$ Total number is less than the sum of the rows because four pairs, in which the two units of the pair exhibited a different type of linkage, were counted twice.

Table 3. Relationship between spike-to-spike correlation, signal correlation, and noise correlation

\begin{tabular}{|c|c|c|c|c|c|c|c|}
\hline \multirow[b]{2}{*}{ Pairs } & \multirow[b]{2}{*}{$n$} & \multicolumn{3}{|c|}{ Signal correlation } & \multicolumn{3}{|c|}{ Noise correlation } \\
\hline & & Mean & Median & SD & Mean & Median & SD \\
\hline All pairs ${ }^{a}$ & 204 & 0.28 & 0.53 & 0.65 & 0.034 & 0.028 & 0.23 \\
\hline Pairs with XCR peak & 16 & 0.34 & 0.61 & 0.63 & -0.023 & -0.0073 & 0.19 \\
\hline Pairs without XCR peak & 65 & 0.38 & 0.64 & 0.60 & 0.015 & 0.028 & 0.24 \\
\hline
\end{tabular}

${ }^{a}$ All pairs of INs for which signal and noise correlation were calculated; spike-triggered averages were calculated for 81 of these.
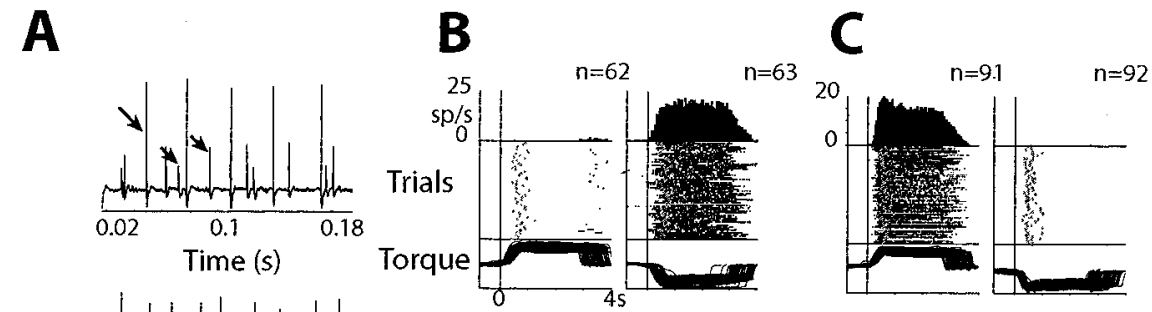

Figure 7. Firing characteristics of SMUs. A, Raw recordings of three SMUs (arrows) from a single electrode (top) and several SMUs from two electrodes (bottom) in single muscles. $B, C$, Responses of an SMU from the extensor carpi radialis muscle $(B)$ and SMU from the flexor carpi radialis muscle (C). Response histograms (top), raster plots (middle), and torque traces (bottom) aligned on torque onset for flexion (left) and extension (right) trials, are shown above autocorrelograms for each SMU. The number of trigger spikes (trigs) and the total time each unit was recorded are given above each autocorrelogram.

area MT (Bair et al., 2001). However, in the spinal cord, noise correlation was independent of both signal correlation and interneuron distance, in contrast to the results from cortex. This independence may reduce the level of noise generated in downstream neurons, such as MNs, which average inputs with similar tuning properties. Independent noise on different pathways will tend to cancel out when these pathways converge on common postsynaptic elements, thereby increasing the accuracy of the transmitted signal (Sompolinsky et al., 2001) (cf. Abbott and Dayan, 1999).

The independence of noise and signal correlation suggests different underlying processes. Signal correlation is likely attributable to common synaptic input from pathways carrying specific, movement-related information, such as corticospinal or muscle receptor axons. IN firing also is affected by various monoaminergic neuromodulators (Jankowska et al., 2000; Schmidt and Jordan, 2000). Because monoamines change excitability without direct activation of the cell, they could induce noise correlation without exerting any signal correlation. Furthermore, monoaminergic fibers appear to have broad termination zones 
Table 4. Correlations between pairs of SMUs

\begin{tabular}{|c|c|c|}
\hline Muscle pair & $n^{a}$ & $\begin{array}{l}\text { Significan } \\
\text { peaks }\end{array}$ \\
\hline \multicolumn{3}{|l|}{ Extensors } \\
\hline$E C R / E C R$ & 10 & 6 \\
\hline $\mathrm{ECU} / \mathrm{ECU}$ & 9 & 2 \\
\hline EDC/EDC & 34 & 17 \\
\hline $\mathrm{ECR} / \mathrm{EDC}$ & 2 & 0 \\
\hline \multicolumn{3}{|l|}{ Flexors } \\
\hline FCR/FCR & 48 & 8 \\
\hline FCU/FCU & 2 & 0 \\
\hline $\mathrm{FCR} / \mathrm{FCU}$ & 15 & 3 \\
\hline FCR/FDS & 2 & 1 \\
\hline \multicolumn{3}{|l|}{ Totals } \\
\hline Pair in same extensor & 53 & 25 \\
\hline Pair in different extensors & 2 & 0 \\
\hline Pair in same flexor & 50 & 8 \\
\hline Pair in different flexors & 17 & 4 \\
\hline All pairs & 122 & 37 \\
\hline
\end{tabular}

ECR, Extensor carpi radialis; ECU, extensor carpi ulnaris; $E D C$, extensor digitorum communis; $F C R$, flexor carpi radialis; $\mathrm{FCU}$, flexor carpi ulnaris; FDS, flexor digitorum superficialis.

${ }^{a}$ Number of pairs for which the $X C R$ had mean counts per bin of $\geq 15$.

(Light, 1985) that could produce noise correlation that is only weakly dependent on distance.

Despite the evidence for common input, we found very little synchronized firing between pairs of simultaneously recorded INs. Central peaks in XCRs of IN activity were infrequent and brief and had very small areas. In contrast, the amplitude of XCR peaks between motor cortical neurons is substantially larger (Lee et al., 1998) (cf. Smith and Fetz, 1989; Baker et al., 2001).

One possible explanation for the weak spike-to-spike correlation is that we recorded primarily from randomly sampled pairs of INs. We did not attempt to find two neurons from the same functional group, which might be expected to receive more common input. However, functionally similar INs do not seem to receive inputs from the same afferents (Harrison and Jankowska, 1985; Edgley, 2001), suggesting that INs located close together are the most likely to receive common input and hence to fire synchronously. In fact, all IN pairs with significant, albeit weak, XCR peaks were recorded from the same electrode. Nonetheless, we did find a higher percentage of correlated firing among pairs of INs with linkages to coactive muscles, and this synchrony was exhibited primarily for the behavioral epoch when those muscles were recruited. Unfortunately, we have data from very few such pairs. It may be that INs with similar output connectivity exhibit more correlated firing than the spinal network in general, as seems to be the case in the motor cortex (Georgopoulos et al., 1993; Jackson et al., 2003).

\section{Correlated firing of SMUs}

Previous studies have shown that SMUs are correlated during voluntary movements. Although direct corticomotoneuronal inputs contribute to these correlations (Baker et al., 1988; Datta et al., 1991; Farmer et al., 1997; Smith et al., 1999), the role of IN synchrony was unclear. In our study, $30 \%$ of SMU pairs fired synchronously, more than double the percentage for IN pairs. In addition, SMUs within single extensor muscles were the most frequently correlated, in contrast with the slight tendency for INs to be correlated during flexion. These data suggest that correlated firing between INs does not contribute significantly to MN synchrony. However, we cannot rule out the possibility that correlated activity between premotor INs of the same muscle contributes to SMU synchrony because we have little data on such pairs. The net effect of a large population of weakly correlated premotor INs also could generate more SMU synchrony than predicted by pairwise correlations.

The extent to which correlated firing between INs contributes to the generation of synchrony effects (i.e., features that straddle the trigger time) in averages of muscle activity triggered by IN discharges (Perlmutter et al., 1998) remains unclear. On one
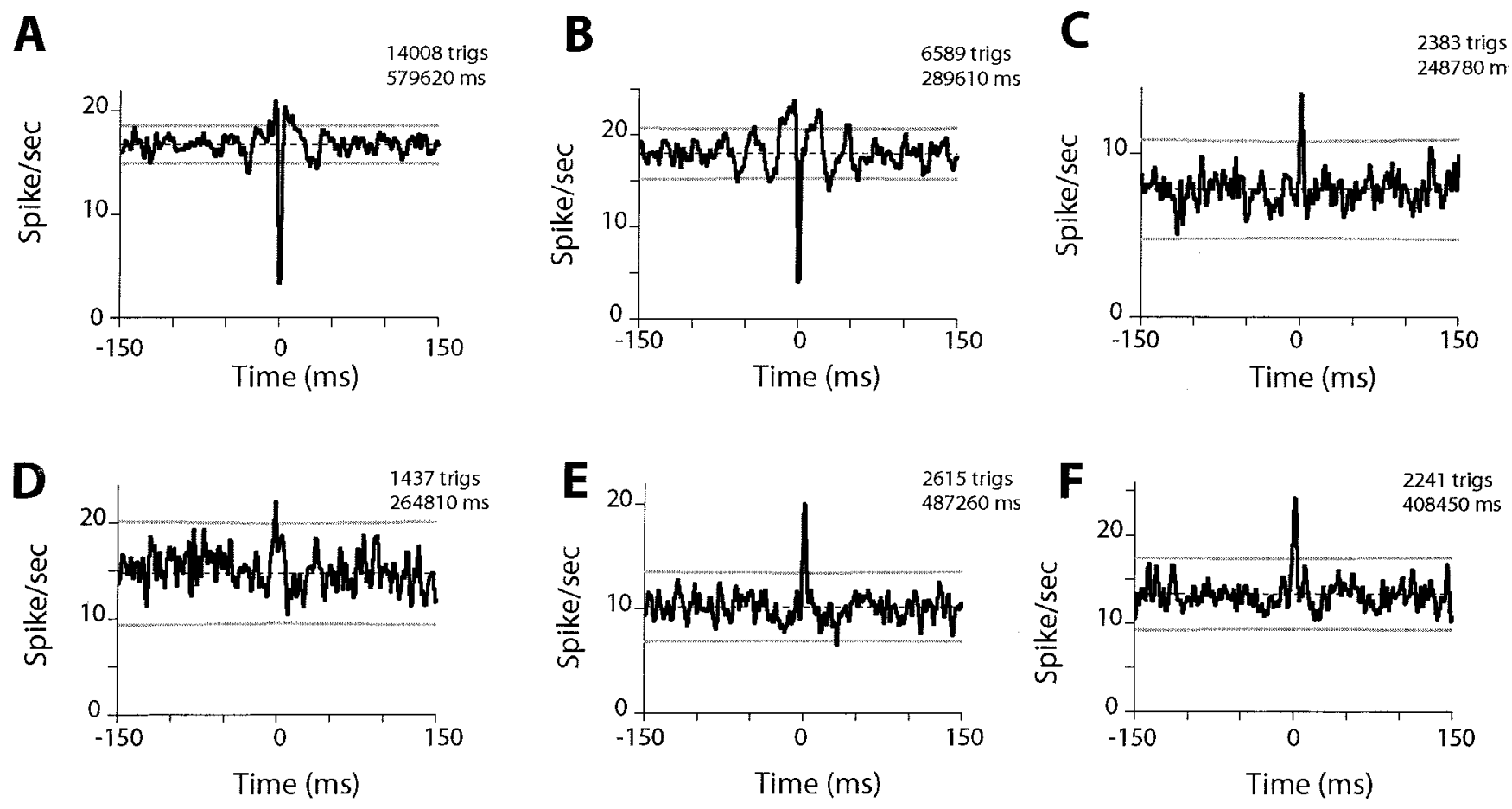

Figure 8. XCRs for six pairs of SMUs $(A-F)$. The number of trigger spikes (trigs) and the total time of recording are shown above each XCR. 
hand, INs with XCR peaks and correlational linkages with muscles tended to be correlated during the behavioral epoch in which the affected muscles were active (i.e., when the synchrony effects were expressed). Also, the duration of these XCR peaks was similar to the time span of synchrony effects produced in forearm muscles. On the other hand, many pairs of INs with correlational linkages with the same or synergistic muscles exhibited no correlated firing. One other factor that could contribute to the generation of synchrony effects is the regular discharge of INs (Prut and Perlmutter, 2003). When spike trains are periodic, the influence of each spike is correlated with effects from the previous spikes.

\section{Decorrelation by spinal interneurons: a hypothesis}

The existence of strong signal correlation and weak spike-tospike correlation between pairs of nearby INs seems paradoxical. It is possible that the common input that produces covariation of activity is too weak to induce synchrony. However, this seems unlikely given existing anatomical evidence on the divergence of descending and somatosensory afferent axons within the cord.

We hypothesize, therefore, that neural activity in the cervical enlargement is actively decorrelated by INs. What could be the purpose of such a computation? We can envision two reasons why correlated activity in spinal premotor networks might be undesirable (Maltenfort et al., 1998).

First, although correlated activity between MNs is a well established feature of normal movement, its relationship to physiological tremor (Erimaki and Christakos, 1999; Halliday et al., 1999) suggests that very high levels of MN synchrony could cause periodic force components that would disrupt motor coordination (Baker et al., 1992). Active decorrelation might act to maintain $\mathrm{MN}$ synchrony at functionally appropriate levels.

Second, correlated activity reduces the information capacity of a neural network (Atick, 1992). This may be functionally important in the cord because of the anatomy of spinal motor circuits. INs appear to be the main source of inputs to MNs, but in each segment, there are far fewer MNs than INs (Gelfan, 1964). Descending pathways to the cord also comprise a larger number of presynaptic than postsynaptic elements. This funneling of neural elements could limit the flexibility or control capabilities of $\mathrm{MN}$ pools unless information is represented as efficiently as possible, in analogy with the information bottleneck proposed for visual pathways (Atick, 1992; Dan et al., 1996). Active decorrelation might act to prevent redundancy in the spinal representation of premotor information, as proposed for the basal ganglia (BarGad et al., 2000).

Our companion paper (Prut and Perlmutter, 2003) reported that INs fire very regularly and suggested that this may reflect uncorrelated activity in the spinal cord, as reported here. An inverse relationship between synchrony and regularity is consistent with previous findings for other types of central neurons (Schulz et al., 1985; Davey and Ellaway, 1988). Nonetheless, the extent to which synchrony, regularity, and the output connectivity of INs are coupled is still unclear. Although INs with correlational linkages with muscles fire more regularly than INs without such linkages, there was no significant difference between the extent of synchrony for these two types of INs. In addition, we found no statistically significant relationship between regularity and correlated firing for the total population of INs. However, some INs with postspike or synchrony effects in muscles were correlated with other neurons only during the epoch in which those muscles were active. This suggests that the synchronization was related in some way to the generation of muscle activity.

The present study does not address the mechanism mediating the active decorrelation of IN activity. Possibilities proposed in other pathways include recurrent inhibition (Maltenfort et al., 1998), presynaptic inhibition that decouples common input (Lomeli et al., 1998), and dynamically modulated lateral inhibition (Bar-Gad et al., 2000).

The results presented in this and the companion paper (Prut and Perlmutter, 2003) extend previous information on the differences between the properties of segmental INs and MNs. INs, with high-gain and dynamically modulated input-output transformations, are suited to rapid translation of inputs into a variety of outputs depending on the behavioral context. Changes in the magnitude or extent of synchronous firing between INs probably do not contribute to this task-dependent flexibility because the spinal network appears to decorrelate inputs that drive MNs. Instead, changes in the temporal firing patterns of individual INs, caused by the activation of different inputs with different firing statistics, may play an important role. These temporal mechanisms can act in concert with other well established processes to modulate transmission through spinal circuitry in a taskdependent manner.

\section{References}

Abbott LF, Dayan P (1999) The effect of correlated variability on the accuracy of a population code. Neural Comput 11:91-101.

Abeles M (1991) Corticonics: neural circuits of the cerebral cortex. Cambridge, UK: Cambridge UP.

Atick JJ (1992) Could information theory provide an ecological theory of sensory processing? Network 3:213-251.

Bair W, Zohary E, Newsome WT (2001) Correlated firing in macaque visual area MT: time scales and relationship to behavior. J Neurosci 21:1676-1697.

Baker JR, Bremner FD, Cole JD, Stephens JA (1988) Short-term synchronization of intrinsic hand muscle motor units in "deafferented" man. J Physiol (Lond) 396:155P.

Baker JR, Davey NJ, Ellaway PH, Friedland CL (1992) Short-term synchrony of motor unit discharge during weak isometric contraction in Parkinson's disease. Brain 115:137-154.

Baker SN, Spinks R, Jackson A, Lemon RN (2001) Synchronization in monkey motor cortex during a precision grip task. I. Task-dependent modulation in single-unit synchrony. J Neurophysiol 85:869-885.

Bar-Gad I, Havazelet-Heimer G, Goldberg JA, Ruppin E, Bergman H (2000) Reinforcement-driven dimensionality reduction: a model for information processing in the basal ganglia. J Basic Clin Physiol Pharmacol 11:305-320.

Brown AG (1981) Organization in the spinal cord. Berlin: Springer. 
Brown PB, Koerber HR, Yezierski RP (1979) Cross-correlation analysis of connectivities among cat lumbosacral dorsal horn cells. J Neurophysiol 42:1199-1211.

Dan Y, Atick JJ, Reid RC (1996) Efficient coding of natural scenes in the lateral geniculate nucleus: experimental test of a computational theory. J Neurosci 16:3351-3362.

Datta AK, Farmer SF, Stephens JA (1991) Central nervous pathways underlying synchronization of human motor unit firing studied during voluntary contractions. J Physiol (Lond) 432:401-425.

Davey NJ, Ellaway PH (1988) Control from the brainstem of synchrony of discharge between gamma motoneurones in the cat. Exp Brain Res 72:249-263.

Eblen-Zajjur AA, Sandkuhler J (1997) Synchronicity of nociceptive and non-nociceptive adjacent neurons in the spinal dorsal horn of the rat: stimulus-induced plasticity. Neuroscience 76:39-54.

Edgley SA (2001) Organisation of inputs to spinal interneurone populations. J Physiol (Lond) 533:51-56.

Erimaki S, Christakos CN (1999) Occurrence of widespread motor-unit firing correlations in muscle contractions: their role in the generation of tremor and time-varying voluntary force. J Neurophysiol 82:2839-2846.

Farmer SF, Halliday DM, Conway BA, Stephens JA, Rosenberg JR (1997) A review of recent applications of cross-correlation methodologies to human motor unit recording. J Neurosci Methods 74:175-187.

Gelfan S (1964) Neuronal interdependence. Prog Brain Res 11:238-260.

Georgopoulos AP, Taira M, Lukashin A (1993) Cognitive neurophysiology of the motor cortex. Science 260:47-52.

Grammont F, Riehle A (1999) Precise spike synchronization in monkey motor cortex involved in preparation for movement. Exp Brain Res 128:118-122.

Halliday DM, Conway BA, Farmer SF, Rosenberg JR (1999) Loadindependent contributions from motor-unit synchronization to human physiological tremor. J Neurophysiol 82:664-675.

Harrison PJ, Jankowska E (1985) Organization of input to the interneurones mediating group I non-reciprocal inhibition of motoneurones in the cat. J Physiol (Lond) 361:403-418.

Hartigan JA (1975) Clustering algorithms. New York: Wiley.

Hatsopoulos NG, Ojakangas CL, Paninski L, Donoghue JP (1998) Information about movement direction obtained from synchronous activity of motor cortical neurons. Proc Natl Acad Sci USA 95:15706-15711.

Ishizuka N, Mannen H, Hongo T, Sasaki S (1979) Trajectory of group Ia afferent fibers stained with horseradish peroxidase in the lumbosacral spinal cord of the cat: three dimensional reconstructions from serial sections. J Comp Neurol 186:189-211.

Jackson A, Gee VJ, Baker SN, Lemon RN (2003) Synchrony between neurons with similar muscle fields in monkey motor cortex. Neuron 38:115-125.

Jankowska E, Hammar I, Chojnicka B, Heden CH (2000) Effects of monoamines on interneurons in four spinal reflex pathways from group I and/or group II muscle afferents. Eur J Neurosci 12:701-714.

Lee D, Port NL, Kruse W, Georgopoulos AP (1998) Variability and correlated noise in the discharge of neurons in motor and parietal areas of the primate cortex. J Neurosci 18:1161-1170.

Li Q, Martin JH (2002) Postnatal development of connectional specificity of corticospinal terminals in the cat. J Comp Neurol 447:57-71.

Light AR (1985) The spinal terminations of single, physiologically characterized axons originating in the pontomedullary raphe of the cat. J Comp Neurol 234:536-548.

Lomeli J, Quevedo J, Linares P, Rudomin P (1998) Local control of information flow in segmental and ascending collaterals of single afferents. Nature 395:600-604.

Maltenfort MG, Heckman CJ, Rymer WZ (1998) Decorrelating actions of
Renshaw interneurons on the firing of spinal motoneurons within a motor nucleus: a simulation study. J Neurophysiol 80:309-323.

Maynard EM, Hatsopoulos NG, Ojakangas CL, Acuna BD, Sanes JN, Normann RA, Donoghue JP (1999) Neuronal interactions improve cortical population coding of movement direction. J Neurosci 19:8083-8093.

Murthy VN, Fetz EE (1996) Synchronization of neurons during local field potential oscillations in sensorimotor cortex of awake monkeys. J Neurophysiol 76:3968-3982.

Oram MW, Hatsopoulos NG, Richmond BJ, Donoghue JP (2001) Excess synchrony in motor cortical neurons provides redundant direction information with that from coarse temporal measures. J Neurophysiol 86:1700-1716.

Perkel DH, Gerstein GL, Moore GP (1967) Neuronal spike trains and stochastic point processes. II. Simultaneous spike trains. Biophys J 7:419-440.

Perlmutter SI, Maier MA, Fetz EE (1998) Activity of spinal interneurons and their effects on forearm muscles during voluntary wrist movements in the monkey. J Neurophysiol 80:2475-2494.

Prut Y, Fetz EE (1999) Primate spinal interneurons show pre-movement instructed delay activity. Nature 401:590-594.

Prut Y, Perlmutter SI (2003) Firing properties of spinal interneurons during voluntary movement. I. State-dependent regularity of firing. J Neurosci 23:9600-9610.

Ralston III HJ, Light AR, Ralston DD, Perl ER (1984) Morphology and synaptic relationships of physiologically identified low-threshold dorsal root axons stained with intra-axonal horseradish peroxidase in the cat and monkey. J Neurophysiol 51:777-792.

Riehle A, Grun S, Diesmann M, Aertsen A (1997) Spike synchronization and rate modulation differentially involved in motor cortical function. Science 278:1950-1953.

Schmidt BJ, Jordan LM (2000) The role of serotonin in reflex modulation and locomotor rhythm production in the mammalian spinal cord. Brain Res Bull 53:689-710.

Schmied A, Ivarsson C, Fetz EE (1993) Short-term synchronization of motor units in human extensor digitorum communis muscle: relation to contractile properties and voluntary control. Exp Brain Res 97:159-172.

Schmied A, Pouget J, Vedel JP (1999) Electromechanical coupling and synchronous firing of single wrist extensor motor units in sporadic amyotrophic lateral sclerosis. Clin Neurophysiol 110:960-974.

Schulz G, Lambertz M, Schulz B, Langhorst P, Krienke B (1985) Reticular formation of the lower brainstem: a common system for cardiorespiratory and somatomotor functions. Cross-correlation analysis of discharge patterns of neighbouring neurones. J Auton Nerv Syst 12:35-62.

Sears TA, Stagg D (1976) Short-term synchronization of intercostal motoneurone activity. J Physiol (Lond) 263:357-381.

Shinoda Y, Yokota J, Futami T (1981) Divergent projection of individual corticospinal axons to motoneurons of multiple muscles in the monkey. Neurosci Lett 23:7-12.

Smith WS, Fetz EE (1989) Effects of synchrony between primate corticomotoneuronal cells on post-spike facilitation of muscles and motor units. Neurosci Lett 96:76-81.

Smith HC, Davey NJ, Savic G, Maskill DW, Ellaway PH, Frankel HL (1999) Motor unit discharge characteristics during voluntary contraction in patients with incomplete spinal cord injury. Exp Physiol 84:1151-1160.

Sompolinsky H, Yoon H, Kang K, Shamir M (2001) Population coding in neuronal systems with correlated noise. Phys Rev E Stat Nonlin Soft Matter Phys 64:051904.

Vaadia E, Haalman I, Abeles M, Bergman H, Prut Y, Slovin H, Aertsen A (1995) Dynamics of neuronal interactions in monkey cortex in relation to behavioural events. Nature 373:515-518.

Windhorst U (1988) How brain like is the spinal cord? Interacting cell assemblies in the nervous system. Berlin: Springer. 